\title{
Experimental Design Optimization of a Sequential Injection Method for Promazine Assay in Bulk and Pharmaceutical Formulations
}

\author{
Abubakr M. Idris, ${ }^{1}$ Fahad N. Assubaie, ${ }^{1}$ and Salah M. Sultan ${ }^{2}$ \\ ${ }^{1}$ Department of Chemistry, King Faisal University, P.O. Box 400, Hofuf 31982, Saudi Arabia \\ ${ }^{2}$ Department of Chemistry, King Fahd University for Petroleum and Minerals, P.O. Box 2026, Dhahran 31261, Saudi Arabia
}

Received 8 May 2007; Accepted 7 June 2007

Experimental design optimization approach was utilized to develop a sequential injection analysis (SIA) method for promazine assay in bulk and pharmaceutical formulations. The method was based on the oxidation of promazine by Ce(IV) in sulfuric acidic media resulting in a spectrophotometrically detectable species at $512 \mathrm{~nm}$. A $3^{3}$ full factorial design and response surface methods were applied to optimize experimental conditions potentially controlling the analysis. The optimum conditions obtained were $1.0 \times 10^{-4} \mathrm{M}$ sulphuric acid, $0.01 \mathrm{M} \mathrm{Ce}(\mathrm{IV})$, and $10 \mu \mathrm{L} / \mathrm{s}$ flow rate. Good analytical parameters were obtained including range of linearity $1-150 \mu \mathrm{g} / \mathrm{mL}$, linearity with correlation coefficient 0.9997 , accuracy with mean recovery $98.2 \%$, repeatability with RSD 1.4\% ( $n=7$ consequent injections), intermediate precision with RSD 2.1\% ( $n=5$ runs over a week), limits of detection $0.34 \mu \mathrm{g} / \mathrm{mL}$, limits of quantification $0.93 \mu \mathrm{g} / \mathrm{mL}$, and sampling frequency 23 samples/h. The obtained results were realized by the British Pharmacopoeia method and comparable results were obtained. The provided SIA method enjoys the advantages of the technique with respect to rapidity, reagent/sample saving, and safety in solution handling and to the environment.

Copyright (c) 2007 Abubakr M. Idris et al. This is an open access article distributed under the Creative Commons Attribution License, which permits unrestricted use, distribution, and reproduction in any medium, provided the original work is properly cited.

\section{INTRODUCTION}

Experimental conditions including chemical and instrumental effect dependently and/or independently on the efficiency of analytical methods in different levels. Therefore, the optimization of these conditions potentially develops analytical methods. Despite its limitation, the univariate method has still been applied for the optimization of analytical methods. This may be due to its simplicity and familiarity. The univariate method optimizes conditions one-by-one by varying levels of one condition while keeping others constant at unspecified levels.

Experimental design, including factorial design and response surface, is a multivariate approach recommended for the development of analytical methods. This approach is applied to (a) reduce large amount of data that could be easily interpreted, (b) examine main and interaction effects of experimental conditions on the efficiency of methods, and (c) optimize simultaneously experimental conditions regarding their interaction with each other by a minimum number of experiments [1]. Furthermore, the response surface method is a powerful tool for ruggedness testing [1]. Rugged- ness evaluates the efficiency of analytical methods under the variation of experimental conditions [2].

In 1990, sequential injection analysis (SIA) technique was introduced as the second generation following flow injection analysis (FIA) technique with dramatic modifications and developments [3]. The versatility of SIA enables the technique to hyphen with different types of detection including spectrophotometry, fluorescence, chemiluminescence, electrochemical, mass spectrometry, and so forth. In addition, as SIA is a fully automated technique, more accurate, precise and safer analytical methods could be adopted; rather than safety in solution handling and reduction of manpower in analytical laboratories. Furthermore, the miniaturization of the technique drastically reduces volumes of reagents/samples from the scale of milliliters to microliters. This does not only offer reagent/sample saving but also offers rapidity and safety to the environment. Due to these advantages, SIA has been extensively applied to pharmaceutical analysis $[4,5]$.

Promazine is chemically known as 3-(10H-phenothiazin-10-yl)- $N, \quad N$-dimethylpropan-1-amine hydrochloride (Figure 2). It is a phenothiazine neuroleptic agent with strong anticholinergic, hypotensive, and sedative effects and 
moderate antiemetic effects. Promazine is additionally used as an adjunct agent in the management of severe pain [6-8].

The increasing use of promazine in medicine has prompted the development of several methods for its quantitative determination in bulk form and pharmaceutical preparations. For this purpose, a wide variety of analytical techniques were utilized including titrimetry $[9,10]$, spectrophotometry $[7,8,11]$, chemiluminescence $[12,13]$, electrophoresis $[14,15]$, conductimetry [16], polarography [17], and FIA $[18,19]$.

In general, most of the previous spectrophotometric methods for phenothiazines assay are unsatisfactory for different reasons, for example, some of them lacked sensitivity or specificity [20-23]. Some others required long time of heating or lengthy procedure or were conducted in nonaqueous media [24]. Moreover, these methods are not straightforward since they did not base on the detection of one of promazine derivatives.

In the present study, SIA technique was utilized to adopt a new method for the assay of promazine hydrochloride in bulk and pharmaceutical preparations. The method was based on the oxidation of promazine by $\mathrm{Ce}(\mathrm{IV})$ in sulphuric acid media resulting in a spectrophotometrically detectable species. Experimental conditions potentially controlling the method including $\mathrm{Ce}(\mathrm{IV})$ concentration, sulfuric acid concentration, and flow rate were optimized using experimental design-based methods.

\section{EXPERIMENTAL}

\subsection{Chemicals and reagents}

All chemicals and reagents used in this study were of analytical reagent grade; and the quality of water was distilled deionized. Promazine hydrochloride was supplied from Sigma (Taufkirchen, Germany). Ammonium cerium sulphate dihydrate $\left(\mathrm{Ce}\left(\mathrm{NH}_{4}\right)_{4}\left(\mathrm{SO}_{4}\right)_{4} \cdot 2 \mathrm{H}_{2} \mathrm{O}\right)$, hydrochloric acid, sulphuric acid, and sodium hydroxide were supplied from Fluka (Buchs, Switzerland). Promazine hydrochloride in bulk form as well as inactive ingredients possibly found in pharmaceutical formulations were a generous gift from Samf (Khartoum North, Sudan). These ingredients included sodium citrate, citric acid, sodium formaldehyde sulphoxylate, microcrystalline cellulose, magnesium stearate, maize starch, titanium dioxide, carnauba wax, propylene glycol, povidone, and talc.

\subsection{Instrumentation and software packages}

The manifold used in this method is composed of sequential injection analyzer combined with miniaturized fiber optic spectrometer. Full components of the manifold are diagrammed in Figure 1.

SIA manifold used in this study is a FIALab 3500 (Medina, WA USA). It is composed of a syringe pump (SP), multiposition valve (MPV), holding coil (HC), and Z-flow cell ( $\mathrm{Z}$ ) as well as pump tubing and personal computer (PC). The SP includes 24,000 increments with high-resolution stepper motor, which drives the piston at rates from 1.5 seconds to $10.0 \mathrm{~min}$ per stroke. It is $>99 \%$ accuracy at full stroke. The syringe has a volume of $2.5 \mathrm{~mL}$. The MPV is chemically inert and has eight ports with a standard pressure of 250 psi (gas)/600 psi (liquid); zero dead volume. The $\mathrm{Z}$ is $10 \mathrm{~mm}$ path-length Plexiglass compatible with fiber optic connectors. Pump tubing of " 0.03 inch" ID Teflon type supplied from Upchurch Scientific, Inc. (Oak Harbor, WA, USA) was used to connect SIA units and to make HC with a long of $200 \mathrm{~cm}$.

The optical devices were composed of radiation source, spectrometer, and fiber optic connectors. They were fabricated by Ocean Optics (Dunedin Florida, USA). The radiation source is an LS-1 Tungsten Halogen lamb optimized for VIS-NIR (360 nm-2 $\mu \mathrm{m}$ wavelength range). The detector is a USB2000 Spectrometer adapted to 200-1100 nm wavelength range. The fiber optic connectors are 200 micron Sub Miniature version A (SMA).

FIALab for Windows version 5.0 supplied from FIAlab (Medina, WA, USA) was used for programming and controlling SIA manifold. SigmaPlot for Windows version 9.01 supplied from Systat Software, Inc. (Point Richmond, CA, USA) was used for data interpolation and constructing surface plots.

\subsection{Preparation of standard solutions and samples}

A $3.516 \times 10^{-3} \mathrm{M}$ promazine hydrochloride $(1000 \mu \mathrm{g} / \mathrm{mL}$ promazine) as a primary standard solution was prepared in aqueous media and stored protected from light. A $0.10 \mathrm{M}$ $\mathrm{Ce}(\mathrm{IV})$ as a primary standard solution was prepared in $0.01 \mathrm{M}$ sulphuric acid media. Secondary standard solutions of promazine, $\mathrm{Ce}(\mathrm{IV})$, and sulphuric acid were prepared by dilution in the appropriate way.

Promazine is taken by patients in injection or tablets formulations $[7,8]$. These formulations with the appropriate inactive ingredients were prepared at our laboratory. Ampoules were synthesized by mixing $50 \mathrm{mg}$ of promazine hydrochloride with other inactive ingredients including sodium citrate, citric acid, and sodium formaldehyde sulphoxylate in a total quantity of $5 \mathrm{mg}$. Tablets were synthesized by mixing $50 \mathrm{mg}$ of promazine hydrochloride and excipients including microcrystalline cellulose, magnesium stearate, maize starch, titanium dioxide, carnauba wax, propylene glycol, povidone, and talc in a total quantity of $10 \mathrm{mg}$. Ingredients of both injection and tablets formulations were dissolved in water to give a final volume of $10 \mathrm{~mL}$. Placebo injection and tablets samples were prepared by mixing the appropriate excipients. All solutions were filtered and the filtrates were used for further analysis.

\subsection{SIA procedure}

A suitable SIA manifold was constructed. As shown in Figure 1, water was linked to the inposition in the SP and to port-1 in the MPV. Sulphuric acid, Ce(IV), and placebo sample were linked to port 2, 3, and 4, respectively. Standards/samples were linked to port 5 to 7 . $Z$ was linked to port 8. An appropriate protocol controlling the SIA procedure was 


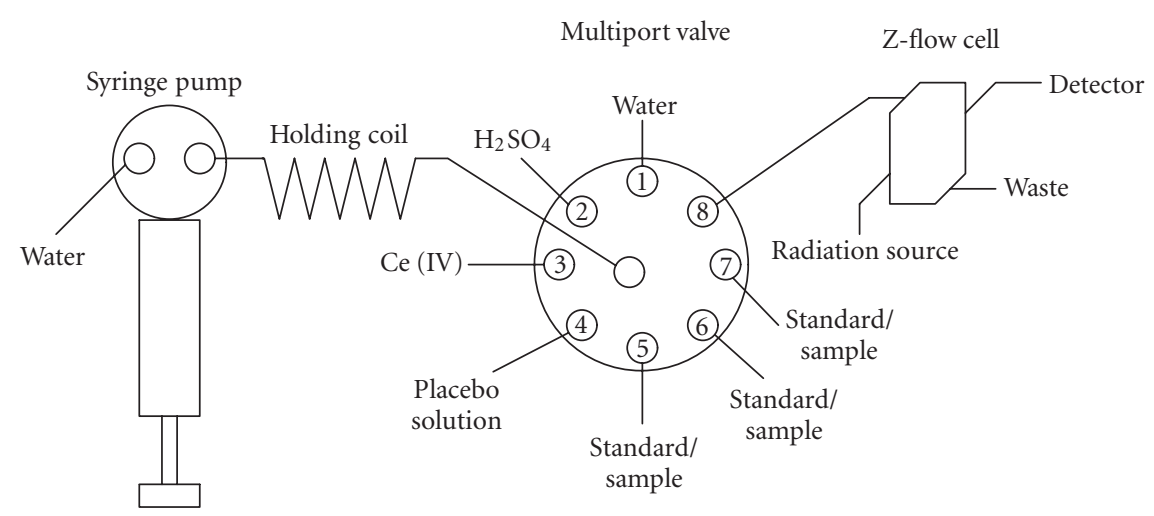

FIGURE 1: Schematic diagram of a SIA manifold constructed for promazine assay.

programmed using FiaLab software. It is briefly described as follows.

(i) At a flow rate of $150 \mu \mathrm{L} / \mathrm{s}$, the syringe was filled with $1000 \mu \mathrm{L}$ of water.

(ii) Tubes were loaded by aspirating $100 \mu \mathrm{L}$ of each of sulphuric acid, $\mathrm{Ce}(\mathrm{IV})$, placebo, and standards/samples, and then the syringe was emptied.

(iii) $30 \mu \mathrm{L}$ of each of sulphuric acid, Ce(IV), and blank solutions and $10 \mu \mathrm{L}$ of water as a spacer solution were sequentially aspirated into the HC. The solutions were mixed by short reverse strokes.

(iv) At the required flow rate, a volume of $1000 \mu \mathrm{L}$ was dispensed to the $\mathrm{Z}$; and the reference and absorbance scan were carried out at wavelength $512 \mathrm{~nm}$.

(v) To measure the absorbance of promazine derivative, steps (iii) and (iv) were repeated with replacing standard/sample solutions instead of placebo solution.

(vi) Values of peak height of absorbance of both placebo and standards/samples were recorded. The difference was calculated and the obtained values are termed as "response" in the following sections.

\section{RESULTS AND DISCUSSION}

\subsection{Preliminary investigation}

A preliminary investigation on a possible oxidation of promazine indicated that promazine is oxidized by the means of $\mathrm{Ce}(\mathrm{IV})$ in sulphuric acid media at room temperature in a fast reaction. The scheme of the reaction is depicted in Figure 2. Similar to what has been earlier proposed to another member of phenothiazines [25], promazine is oxidized to form mono- and dication radicals in two steps. The free radical recorded maximum absorbance at $512 \mathrm{~nm}$, and no spectrum interference was recorded from other species in the matrix of the adopted reaction.

\subsection{Experimental design optimization}

Before undertaking any optimization study, it is important to delineate clearly the boundaries of conditions controlling the analysis, namely, sulphuric acid concentration, $\mathrm{Ce}(\mathrm{IV})$ con-
Promazine structure

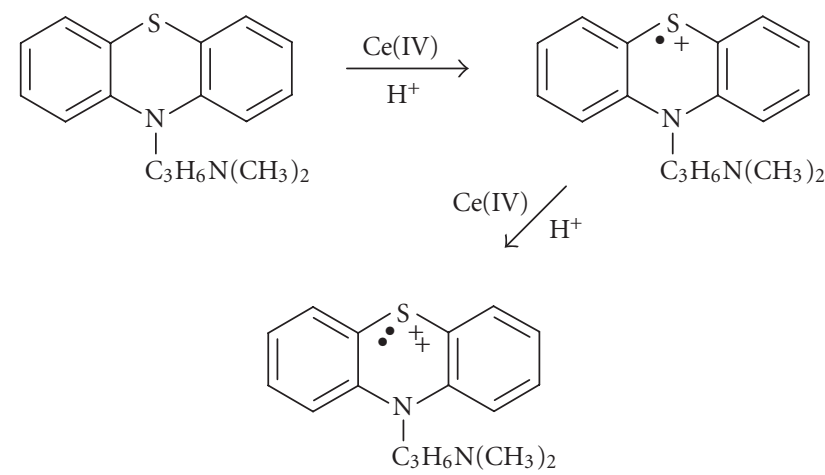

FIGURE 2: Proposed reaction scheme of promazine oxidation by $\mathrm{Ce}(\mathrm{IV})$ in sulphuric acid media.

centration, and flow rate. Preliminary investigation revealed that acid concentration bellow $1.0 \times 10^{-4} \mathrm{M}$, hydrolysis of $\mathrm{Ce}(\mathrm{IV})$, took place. Acid concentration above $0.10 \mathrm{M}$, dense yellow color, was produced in the mixture of $\mathrm{Ce}(\mathrm{IV})$ and the acid. This caused spectrum interference with the analyte. On the other hand, high acid concentration decreased the repeatability of SIA measurement. For $\mathrm{Ce}(\mathrm{IV})$ concentration, $0.01 \mathrm{M}$ was considered at the maximum since beyond this level the solution is not stable. Below $1.0 \times 10^{-4} \mathrm{M}$, the molar equivalency of $\mathrm{Ce}(\mathrm{IV})$ would not be enough to oxidize relatively high concentration of promazine in bulk and pharmaceutical formulations. A flow rate ranging from 10 to $60 \mu \mathrm{L} / \mathrm{s}$ was found to be suitable for spectrophotometric measurement.

When applying experimental design methodologies, it is advisable to keep the number of variables as low as possible in order to avoid very complex response models and large variability [1].

\subsection{The main and interaction effect factors}

A $3^{3}$ full factorial design was carried out; where the base 3 stands for variable levels considering the lowest, the medium, and the highest values; and the power 3 is the number of 
parameters that would be optimized. The ranges obtained from the preliminary investigation were considered as minimum and maximum levels, while medium levels were mathematically calculated. 27 experiments, as the result of the adopted factorial design, were conducted and the results obtained are introduced in Table 1.

The main and interaction effect factors were calculated and the results obtained are depicted in Figure 3. For the main factors, the level of the positive effect of $\mathrm{Ce}$ (IV) concentration was higher than the level of the negative effect of flow rate; and the latter was higher than the level of the negative effect of acid concentration. For the interaction effect factors, the level of the negative interaction effect of $\mathrm{Ce}$ (IV) concentration with flow rate was higher than other levels of interaction effect factors. The effect factor study concluded that $\mathrm{Ce}$ (IV) and flow rate were found to be critically controlling the adopted method. The negative interaction effect of $\mathrm{Ce}(\mathrm{IV})$ concentration with acid concentration may be attributed to the increase of the potential oxidation of $\mathrm{Ce}$ (IV) concentration with the decrease of acid concentration. On the other hand, higher acid concentration negatively effects the stability of the oxidized form of promazine leading to its disassociation. As proposed before, although the oxidation of promazine is fast, the negative effect of flow rate may be due to phenomena that low flow rate delays solution in tubings, and thus dispersion is increased. This enhanced the reaction, and thus the absorbance of the detectable species increased.

\subsection{Response surface}

The coded levels of the adopted factorial design with their respective responses were interpolated; and the response surface plots were constructed. As examples, two figures are depicted. Figure 4 shows the response surface plot as a function of $\mathrm{Ce}(\mathrm{IV})$ concentration versus acid concentration. The highest response obtained was above 0.50 . The general trend of the figure is that the effect of $\mathrm{Ce}(\mathrm{IV})$ concentration is higher than the effect of acid concentration. The bimodal shape appearing at the left side indicates that acid concentration interacts with higher $\mathrm{Ce}(\mathrm{IV})$ concentrations. The response of the surface plot of acid concentration versus flow rate reaches 0.75 . The flow rate resembled a higher effect on the response value than that of acid concentration. Figure 5 shows the response surface plot as a function of $\mathrm{Ce}$ (IV) concentration versus flow rate. As shown in this figure, the highest response obtained was above 1.0 when $\mathrm{Ce}(\mathrm{IV})$ concentration was at the highest level and the flow rate was at the lowest. Therefore, it was decided to consider $0.01 \mathrm{M} \mathrm{Ce}(\mathrm{IV})$ and flow arte $10 \mu \mathrm{L} / \mathrm{s}$ as the optimum. On the other hand, based on its negative effect on the response, $1.0 \times 10^{-4} \mathrm{M}$ was considered as the optimum acid concentration.

\subsection{Method validation}

A long series of standard solutions of promazine were subjected to the optimized SIA method for the purpose of calibration. Beer's law was found to be obeyed in the concentration range of $1-150 \mu \mathrm{g} / \mathrm{mL}$ with weighed regression " $R=$ $0.0082 C+0.0956$," where $R$ is the response, $C$ is the concen-
TABLE 1: A $3^{3}$ factorial design matrix with experimental results (responses).

\begin{tabular}{|c|c|c|c|c|}
\hline \multirow{2}{*}{ Experiment number } & \multicolumn{3}{|c|}{ Factor } & \multirow{2}{*}{ Response } \\
\hline & $\mathrm{A}^{1}$ & $\mathrm{C}^{2}$ & $\mathrm{~F}^{3}$ & \\
\hline 1 & -1 & -1 & -1 & 0.149 \\
\hline 2 & -1 & -1 & 0 & 0.116 \\
\hline 3 & -1 & -1 & +1 & 0.081 \\
\hline 4 & -1 & 0 & -1 & 0.267 \\
\hline 5 & -1 & 0 & 0 & 0.117 \\
\hline 6 & -1 & 0 & +1 & 0.103 \\
\hline 7 & -1 & +1 & -1 & 2.013 \\
\hline 8 & -1 & +1 & 0 & 0.669 \\
\hline 9 & -1 & +1 & +1 & 0.641 \\
\hline 10 & 0 & -1 & -1 & 0.237 \\
\hline 11 & 0 & -1 & 0 & 0.204 \\
\hline 12 & 0 & -1 & +1 & 0.12 \\
\hline 13 & 0 & 0 & -1 & 0.165 \\
\hline 14 & 0 & 0 & 0 & 0.104 \\
\hline 15 & 0 & 0 & +1 & 0.108 \\
\hline 16 & 0 & +1 & -1 & 1.100 \\
\hline 17 & 0 & +1 & 0 & 0.273 \\
\hline 18 & 0 & +1 & +1 & 0.173 \\
\hline 19 & +1 & -1 & -1 & 0.257 \\
\hline 20 & +1 & -1 & 0 & 0.187 \\
\hline 21 & +1 & -1 & +1 & 0.114 \\
\hline 22 & +1 & 0 & -1 & 0.251 \\
\hline 23 & +1 & 0 & 0 & 0.100 \\
\hline 24 & +1 & 0 & +1 & 0.090 \\
\hline 25 & +1 & +1 & -1 & 1.334 \\
\hline 26 & +1 & +1 & 0 & 0.321 \\
\hline 27 & +1 & +1 & +1 & 0.203 \\
\hline
\end{tabular}

(1) Sulphuric acid concentration (M); (2) Ce(IV) concentration (M); (3) flow rate $(\mu \mathrm{L} / \mathrm{s})$.

TABLE 2: Results obtained by the SIA and BP methods for promazine assay in pharmaceutical formulations.

\begin{tabular}{|c|c|c|c|}
\hline \multirow{2}{*}{ Sample type } & \multicolumn{2}{|c|}{ Mean recovery $\pm \mathrm{RSD}^{1}$} & \multirow{2}{*}{ t-test value } \\
\hline & SIA method & BP method & \\
\hline Bulk & $99.4 \pm 0.9$ & $99.1 \pm 1.2$ & 1.4 \\
\hline Injection & $98.1 \pm 1.7$ & $98.6 \pm 2.2$ & 1.7 \\
\hline Tablets & $97.8 \pm 1.6$ & $102.7 \pm 2.4$ & 2.1 \\
\hline
\end{tabular}

(1) Relative standard deviation for 10 consequent injections.

tration of promazine in $\mu \mathrm{g} / \mathrm{mL}$. The correlation coefficient was 0.9997 indicating good linearity. Figure 6 shows a typical SIA calibration obtained under the optimum conditions by triplicate consequent injection of four standard solutions of promazine $(1,50,100$, and $150 \mu \mathrm{g} / \mathrm{mL})$.

The accuracy was examined by analyzing bulk, tablets, and injection formulations. The obtained results were realized by the British Pharmacopoeia (BP) method. BP provided a classical potentiometric titration method by sodium hydroxide for promazine assay in bulk form [7]; and a 


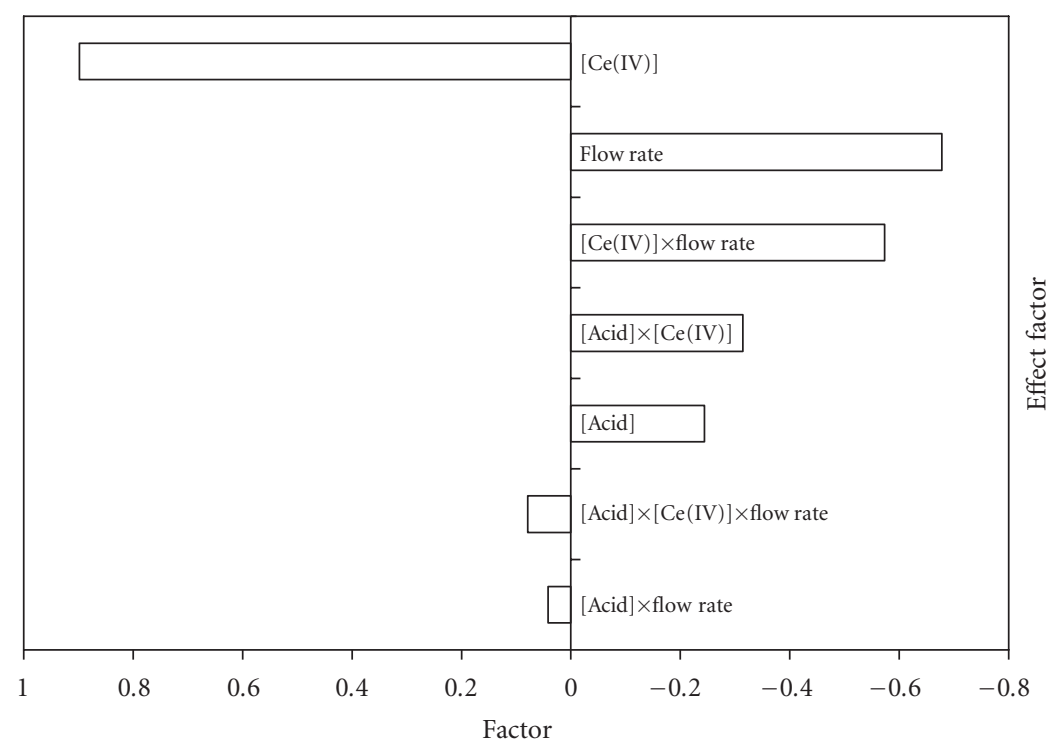

FIGURE 3: The main and interaction effect factors of Ce(IV) concentration (M), acid concentration (M), and flow rate ( $\mu \mathrm{L} / \mathrm{s})$ on the response of the proposed SIA method.

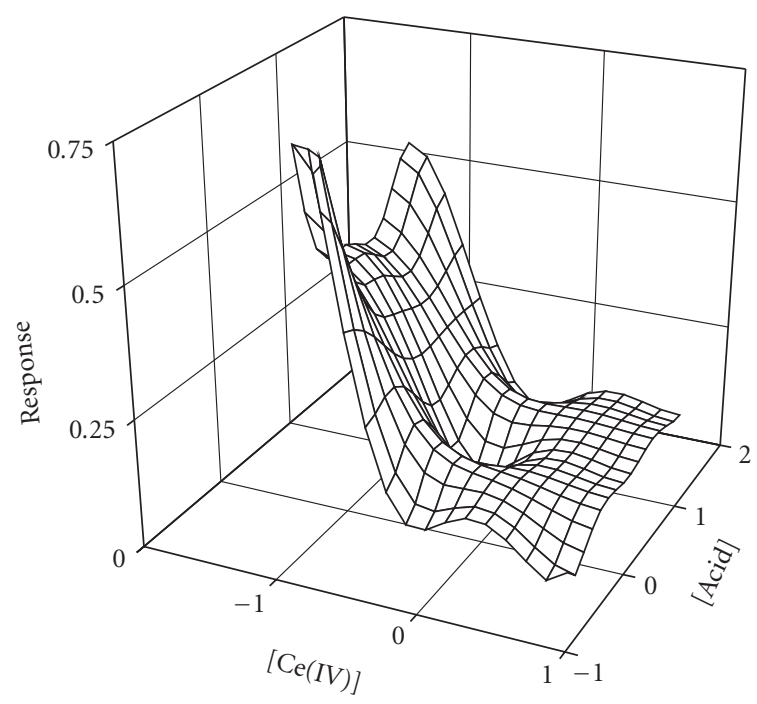

FIGURE 4: Response surface plot of Ce(IV) concentration (M) versus sulphuric acid concentration (M).

classical spectrophotometric method in hydrochloric acid media in tablets and injection formulations. Analysis for each sample was repeated seven times, and the relative standard deviation (RSD) was calculated. The t-test values were also calculated. The results obtained are introduced in Table 2. The obtained results indicating that the provided SIA method is accurate and repeatable.

The intermediate precision of the SIA method was examined by analyzing the same solutions 5 times over a week. Relative standard deviation (RSD) of the mean recovery for samples under study was $2.1 \%$ indicating good intermediate precision.

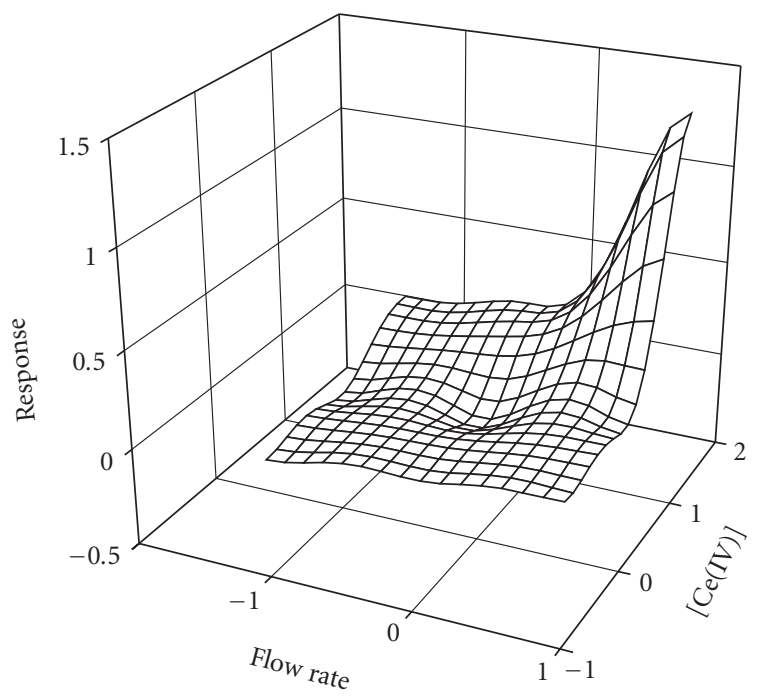

FIGURE 5: Response surface plot of Ce(IV) concentration (M) versus flow rate $(\mu \mathrm{L} / \mathrm{s})$.

The limits of detection (LOD) and quantification (LOQ) were also examined. LOD was calculated as $3.3(\mathrm{~s} / \mathrm{S})$ and LOQ as $10(\mathrm{~s} / \mathrm{S})$ where $\mathrm{s}$ is the standard deviation for seven replicates of the measurement of placebo solution, $S$ is slope of the weighed regression of calibration equation. The LOD and LOQ obtained were 0.34 and $0.93 \mu \mathrm{g} / \mathrm{mL}$, respectively, indicating good detectability.

\section{CONCLUSIONS}

The SIA technique was utilized to adopt a simple, accurate, precise, rapid, and reagent/sample saving method for 


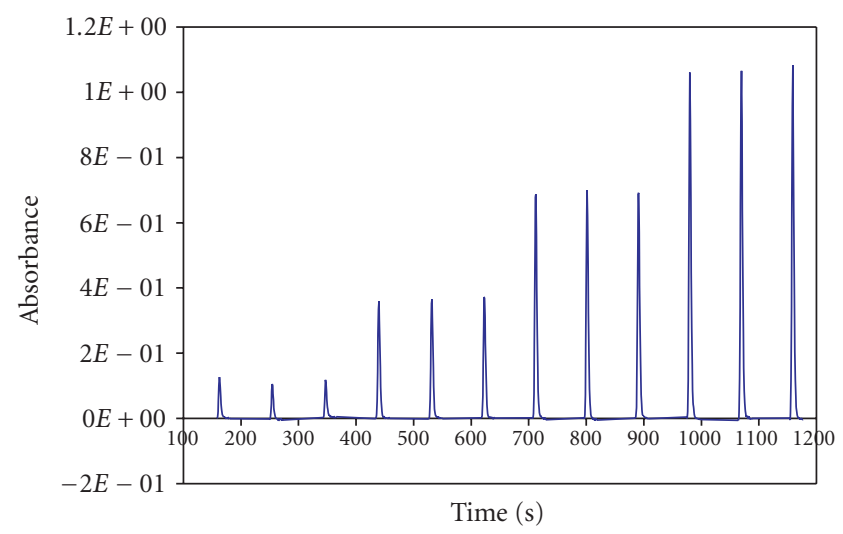

FIGURE 6: A typical SIA calibration obtained by triplicate injection of four standard solutions of promazine $(1,50,100$, and $150 \mu \mathrm{g} / \mathrm{mL})$ under the optimum conditions $30 \mu \mathrm{L}$ of $1.0 \times 10^{-4} \mathrm{M}$ sulphuric acid, $30 \mu \mathrm{L}$ of $0.01 \mathrm{M} \mathrm{Ce}(\mathrm{IV}), 30 \mu \mathrm{L}$ of promazine, $10 \mu \mathrm{L}$ water, and $10 \mu \mathrm{L} / \mathrm{s}$ flow rate.

the assay of promazine in bulk and pharmaceutical formulations. Unlike previous spectrophotometric methods, the current method has the advantage of the straightforwardness for monitoring the actual radical species form of the analyte. The factorial design and response surface methods were successfully applied to optimize experimental conditions controlling the method. This offers a wide range of linearity and good detectability. The merits of automation and miniaturization of the utilized technique rendered the proposed method to have advantages over the BP method with respect to repeatability, rapidity, reagent/sample saving, and safety in solution handling and to the environment.

\section{ACKNOWLEDGMENTS}

This work was supported by the grants from the Deanship of Scientific Research, King Faisal University, Saudi Arabia (Project no. 7049). The financial contribution is gratefully acknowledged. The authors also thank the Department of Chemistry, College of Science, King Faisal University for allowing them to conduct this research in their laboratories. Thanks are also due to Samf (Khartoum North, Sudan) for supplying some materials.

\section{REFERENCES}

[1] E. D. Morgan, Chemometrics: Experimental Design, ACOL, John Wiley \& Sons, London, UK, 1991.

[2] G. Currell, Analytical Instrumentation, Performance Characteristics and Quality, Analytical Techniques in the Sciences, John Wiley \& Sons, New York, NY, USA, 2000.

[3] J. Ruzicka and G. D. Marshall, "Sequential injection: a new concept for chemical sensors, process analysis and laboratory assays," Analytica Chimica Acta, vol. 237, no. 2, pp. 329-343, 1990.

[4] A. M. Pimenta, M. C. B. S. M. Montenegro, A. N. Araújo, and J. M. Calatayud, "Application of sequential injection analysis to pharmaceutical analysis," Journal of Pharmaceutical and Biomedical Analysis, vol. 40, no. 1, pp. 16-34, 2006.
[5] P. D. Tzanavaras and D. G. Themelis, "Review of recent applications of flow injection spectrophotometry to pharmaceutical analysis," Analytica Chimica Acta, vol. 588, no. 1, pp. 1-9, 2007.

[6] V. Larsimont, J. Meins, H. Fieger-Büschges, and H. Blume, "Validated high-performance liquid chromatographic assay for the determination of promazine in human plasma: application to pharmacokinetic studies," Journal of Chromatography B, vol. 719, no. 1-2, pp. 222-226, 1998.

[7] British Pharmacopoeia, E-copy on CD, Version 6.0, 2002.

[8] United States Pharmacopoeia, E-copy on CD, Version 26 NF21, 2003.

[9] A. S. Issa and M. S. Mahrous, "Titrimetric determination of some phenothiazine derivatives, with ferricyanide," Talanta, vol. 31, no. 4, pp. 287-288, 1984.

[10] K. Basavaiah and G. Krishnamurthy, "Oxidimetric titration of some phenothiazine neuroleptics and antiallergics with potassium dichromate," Analytical Sciences, vol. 15, no. 1, pp. 67-71, 1999.

[11] J. Karpińska, "Simultaneous quantification of promazine hydrochloride and its sulfoxide in pharmaceutical preparations," Analytical Sciences, vol. 17, no. 2, pp. 249-253, 2001.

[12] J. Cepas, M. Silva, and D. Pérez-Bendito, "Improved peroxyoxalate chemiluminescence-based determinations by use of continuous reagent addition to remove background emission," Analytical Chemistry, vol. 66, no. 22, pp. 4079-4084, 1994.

[13] G. M. Greenway and S. J. L. Dolman, "Analysis of tricyclic antidepressants using electrogenerated chemiluminescence," Analyst, vol. 124, no. 5, pp. 759-762, 1999.

[14] P. G. H. M. Muijselaar, H. A. Claessens, and C. A. Cramers, "Determination of structurally related phenothiazines by capillary zone electrophoresis and micellar electrokinetic chromatography," Journal of Chromatography A, vol. 735, no. 1-2, pp. 395-402, 1996.

[15] F. J. Lara, A. M. García-Campana, F. Alés-Barrero, and J. M. Bosque-Sendra, "Determination of thiazinamium, promazine and promethazine in pharmaceutical formulations using a CZE method," Analytica Chimica Acta, vol. 535, no. 1-2, pp. 101-108, 2005.

[16] Y. M. Issa, W. F. El-Hawary, and A. F. Ahmed, "Ion-pair formation in pharmaceutical analysis. Conductimetric determination of promazine, chlorpromazine, promethazine, imipramine and ciprofloxacin hydrochlorides in pure form, drug formulations and urine," Mikrochimica Acta, vol. 134, no. 1-2, pp. 9-14, 2000.

[17] F. Belal, S. M. El-Ashry, I. M. Shehata, M. A. El-Sherbeny, and D. T. El-Sherbeny, "Differential-pulse polarographic determination of some $\mathrm{N}$-substituted phenothiazine derivatives in dosage forms and urine through treatment with nitrous acid," Mikrochimica Acta, vol. 135, no. 3-4, pp. 147-154, 2000.

[18] A. Kojlo, H. Puzanowska-Tarasiewicz, and J. Martinez Calatayud, "Spectrophotometric determination of promazine with an oxidative column in FIA manifolds," Journal of Pharmaceutical and Biomedical Analysis, vol. 10, no. 10-12, pp. 785-788, 1992.

[19] H. Puzanowska-Tarasiewicz, E. Wołyniec, and A. Kojło, "Flow injection spectrophotometric determination of promazine," Journal of Pharmaceutical and Biomedical Analysis, vol. 14, no. 3, pp. 267-271, 1996.

[20] E. M. M. Kassem, M. M. Kamel, A. A. Makhlouf, and M. T. Omar, "New 4-substituted phthalazinone derivatives with possible antibacterial activity," Pharmazie, vol. 44, no. 1, pp. 62-63, 1989. 
[21] C. S. P. Sastry, A. S. R. Prasad Tipirneni, and M. V. Suryanarayana, "Spectrophotometric determination of some antiallergic agents with 3-methyl-2-benzothiazolinone hydrazone," Journal of Pharmaceutical and Biomedical Analysis, vol. 8, no. 3, pp. 287-292, 1990.

[22] H. D. Revanasiddappa and P. G. Ramappa, "Spectrophotometric determinations of some phenothiazine drugs," Talanta, vol. 43, no. 8, pp. 1291-1296, 1996.

[23] D. F. Gurka, R. E. Kolinski, J. W. Myrick, and C. E. Wells, "Scope of differential UV and differential fluorescence assays for phenothiazines: comparison with official methods," Journal of Pharmaceutical Sciences, vol. 69, no. 9, pp. 1069-1074, 1980.

[24] M. V. D. Jayarama, H. S. Yathirajan, and Rangaswamy, "Interaction of phenothiazines with nitroso-R salt and extractive spectrophotometric determination of phenothiazine drugs," Talanta, vol. 33, no. 4, pp. 352-354, 1986.

[25] S. M. Sultan, M. O. Hamad Al-Turabi, and J. S. Hwang, "Electron spin resonance for quantitative assay of chlorpromazine in drug formulations by oxidation with cerium(IV) in sulfuric acid media," Talanta, vol. 51, no. 2, pp. 327-331, 2000. 


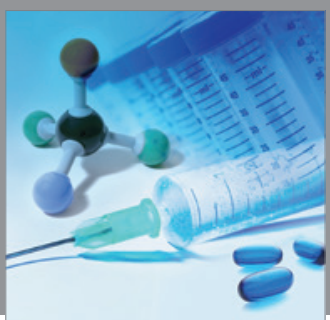

International Journal of

Medicinal Chemistry

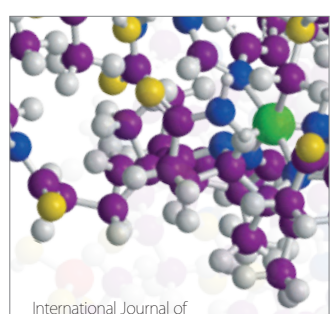

Carbohydrate Chemistry

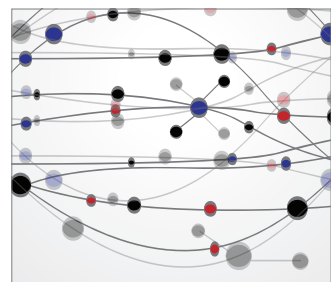

The Scientific World Journal
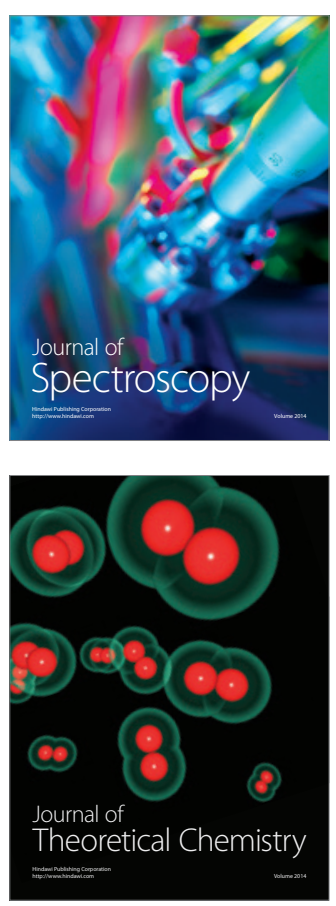
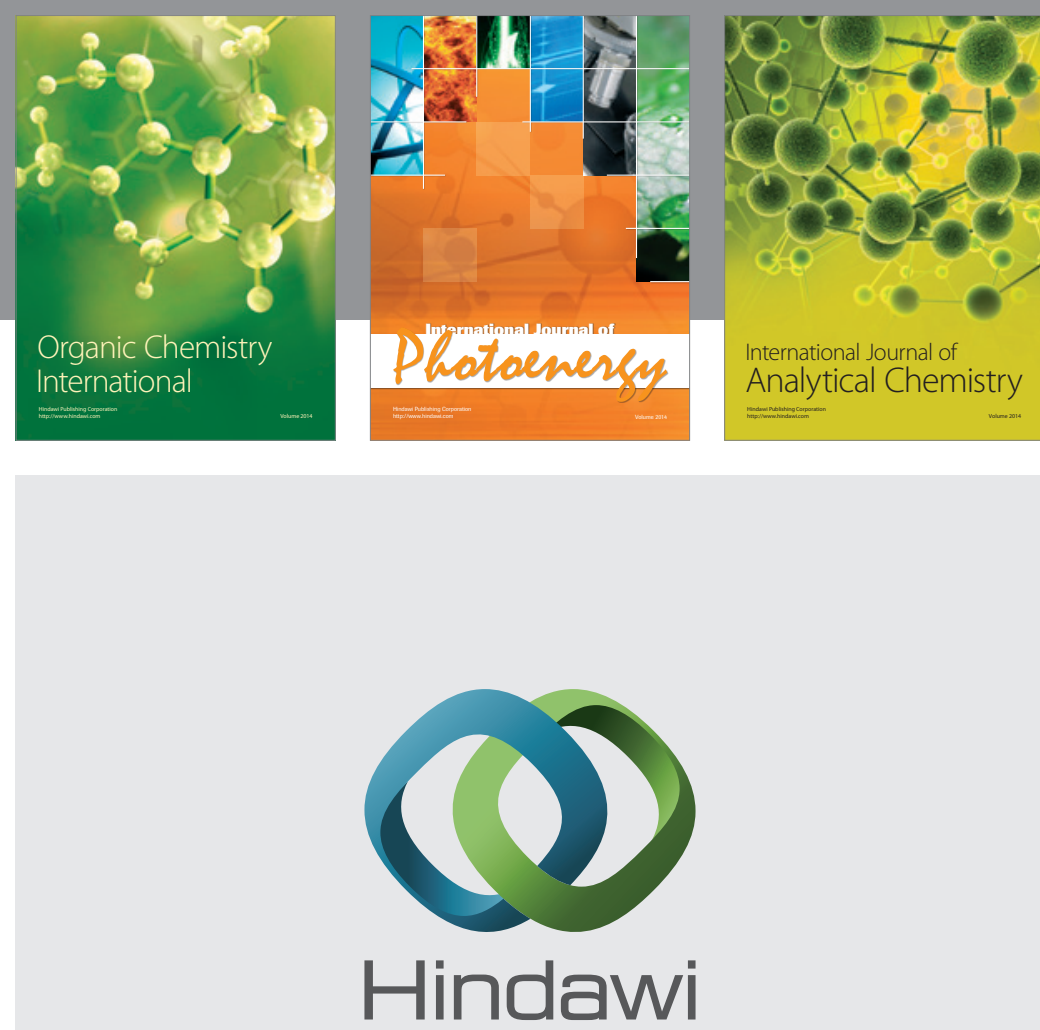

Submit your manuscripts at

http://www.hindawi.com
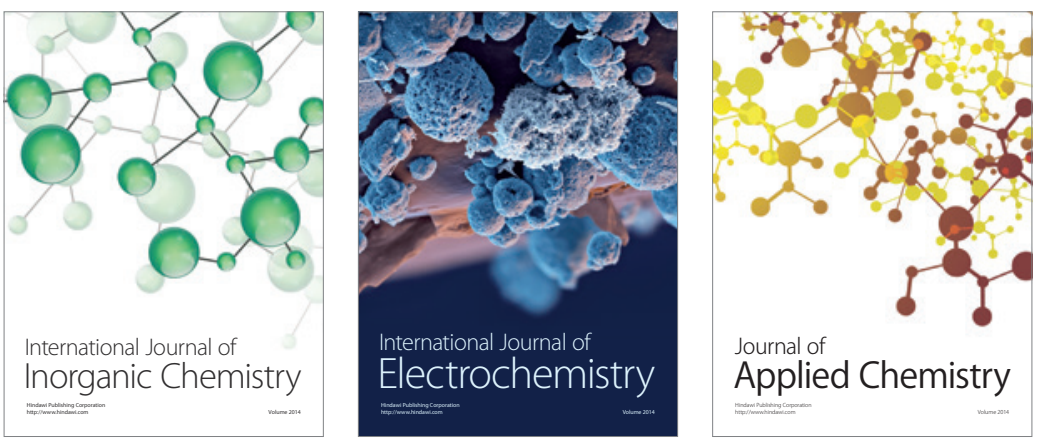

Journal of

Applied Chemistry
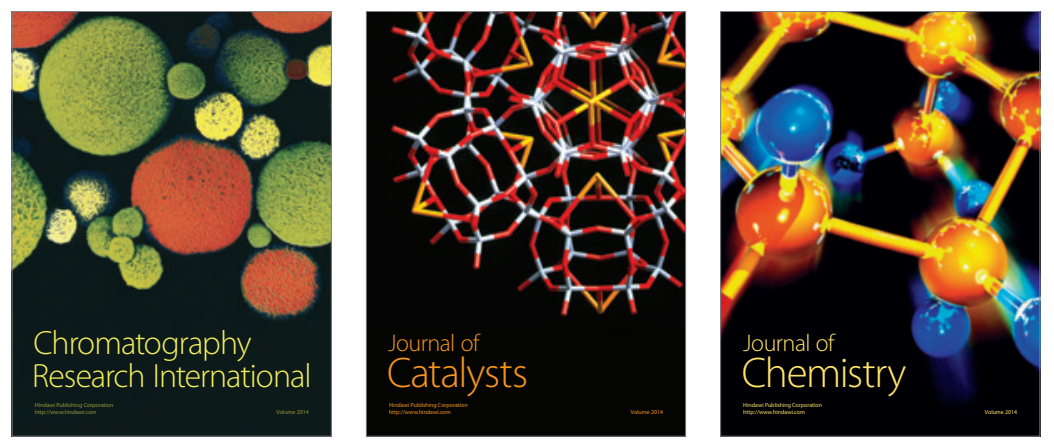
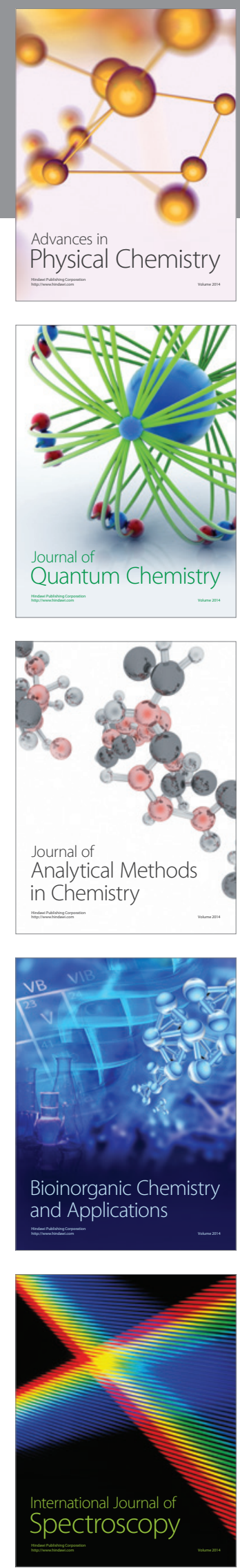